УДК 535; 621.373

DOI 10.25205/2541-9447-2018-13-1-102-107

\author{
А. А. Ковалёв \\ Институт физики полупроводников им. А. В. Ржанова СО РАН \\ пр. Академика Лаврентьева, 13, Новосибирск, 630090, Россия \\ kovalev@isp.nsc.ru

\section{СЕЛЕКТОР ЛИНИЙ ГЕНЕРАЦИИ МОЛЕКУЛЯРНЫХ ЛАЗЕРОВ ИНФРАКРАСНОГО ДИАПАЗОНА}

\begin{abstract}
Исследована применимость методов селекции линий генерации в молекулярных ИК лазерах: помещение дифракционной решетки вместо одного из зеркал резонатора, брэгговского зеркала, наклонного и отражательного интерферометров. Предложен селектор, сочетающий преимущества интерферометра Майкельсона и фазового отражательного интерферометра и позволяющий надежно выделять отдельную колебательно-вращательную линию генерации. Расчет селектора выполнен для случая волноводного $\mathrm{CO}_{2}$ лазера. Проанализированы варианты схем перестройки лазера по линиям генерации при использовании этого селектора.

Ключевые слова: селекция линий генерации в молекулярных ИК лазерах, интерферометр Майкельсона, фазовый отражательный интерферометр.
\end{abstract}

\section{Введение}

Молекулярные лазеры инфракрасного диапазона спектра работают на колебательно-вращательных переходах малых (менее 4 атомов) молекул, таких как $\mathrm{CO}_{2}, \mathrm{CO}, \mathrm{HF}$ и т. п. Среди них наиболее известны лазеры на молекуле двуокиси углерода $\mathrm{CO}_{2}$. Уже в первой публикации [1] были показаны уникальные свойства этого непрерывного газоразрядного лазера, состоящие в относительной простоте конструкции при высокой эффективности генерации когерентного излучения 10-микронного диапазона спектра, представляющего интерес для дистанционного зондирования и многих других фундаментальных и практических приложений.

Сегодня на базе $\mathrm{CO}_{2}$ лазеров - как непрерывных, так и импульсных, как на разреженных газовых смесях, так и на рабочих смесях высокого давления, - создаются мощные системы [2] различного назначения. Область приложений $\mathrm{CO}_{2}$ лазеров простирается от астрофизики до медицины, от систем обработки материалов, источников накачки для получения ВУФ излучения до генерации суперконтинуума в области длин волн 2-40 микрон [3].

Непрерывные газоразрядные $\mathrm{CO}_{2}$ лазеры среднего (порядка 100 Торр) и низкого (десятки Торр) давления, в том числе лазеры на целой серии изотопов $\mathrm{CO}_{2}$, представляют собой ценный инструмент прецизионной спектроскопии. В таких приложениях, как правило, необходимо иметь одночастотное одномодовое излучение, обладающее высокой стабильностью частоты. Проблема надежного выделения генерации на отдельном колебательно-вращательном переходе молекул представляется далекой от окончательного решения. Это связано с тем, что интенсивности линий на соседних по спектру колебательно-вращательных переходах сравнимы по амплитуде, и выделение одной линии требует сильного подавления генерации на ближайших соседних линиях.

В данной работе предложен новый метод селекции колебательно-вращательных пере-

Ковалёв $A$. А. Селектор линий генерации молекулярных лазеров инфракрасного диапазона // Сибирский физический журнал. 2018. Т. 13, № 1. С. 102-107. 
ходов в генерации молекулярного лазера ИК диапазона, сочетающий особенности интерферометра Майкельсона и отражательного интерферометра. Численные расчеты выполнены для случая $\mathrm{CO}_{2}$ лазера низкого или среднего давления рабочей смеси.

\section{Анализ существующих методов выделения линий генерации молекулярных ИК лазеров на основе частотных селекторов}

Методы спектральной селекции продольных мод резонаторов при межмодовых расстояниях 100-1000 МГц подробно рассматривались в [4; 5] преимущественно для газовых лазеров. Выделение спектральных участков шириной около 100 МГц, соответствующих линии генерации, из более широкой спектральной области (например, выделение линии $\mathrm{CO}_{2}$ лазера из области от 9 до 11 микрон), является более сложной задачей.

Для надежного выделения заданной колебательно-вращательной линии излучения молекулярного ИК лазера из всего набора линий генерации (как правило, многочисленных и занимающих большой спектральный диапазон) необходимо выполнить два условия. Во-первых, следует ввести канал потерь для всех нежелательных участков спектра, тем самым подавив генерацию на всех линиях, соседствующих с выделяемой колебательно-вращательной линией. Во-вторых, обеспечить минимальность потерь, вносимых частотным селектором в область генерации выделяемой линии, что позволит обеспечить максимальную выходную мощность одночастотного излучения.

Наиболее распространенный подход состоит в замене одного из зеркал резонатора дифракционной решеткой, работающей в первом порядке дифракции. Обычно используются решетки с периодом 100-150 штр./мм, обеспечивающие в лучшем случае отражение для выделяемой линии на уровне 9095 \%. При невысоком усилении лазера удается этим способом выделить отдельную колебательно-вращательную линию. При более высоком усилении лазер начинает работать на двух-трех соседних линиях генерации, что отчетливо видно по контуру генерации в пределах межмодового расстояния. В работе [6] подробно исследовались свойства такого селектора для волно- водного $\mathrm{CO}_{2}$ лазера (диаметр волновода из окиси бериллия 2,5 мм; активная длина 20 см, решетка 150 штр./мм, расстояние от торца волновода до решетки 18 мм) на примере линии генерации 10P(22). Для этого селектора канал потерь для подавляемых линий обеспечен выходом волны из резонатора за счет угловой дисперсии решетки. В работе [6] было показано экспериментально, что потери, вносимые дифракционной решеткой, составляют $7 \%$ для выделяемой линии генерации. Для ближайших соседних линий (справа и слева от выделяемой) потери составили 10-11\%, а для следующей пары линий - 22-25\%. В то же время для надежного выделения отдельной линии генерации необходимо внести потери $\geq 30 \%$ для пары ближайших соседних линий, т. е. коэффициент отражения для подавляемых линий должен быть 0,7. Далее, для определенности, будем ориентироваться на это значение, задающее необходимую селективность по спектру. Расчет селекции линий $\mathrm{CO}_{2}$ лазера для случая дифракционной решетки, расположенной у края волновода, проведен в [7].

В качестве альтернативы дифракционной решетке можно рассмотреть брэгговское зеркало, состоящее из чередующихся слоев с высоким и низким показателями преломления (например, $n_{H}=3,3$ GaAs и $n_{L}=$ $=2,4 \mathrm{ZnSe})$ с толщинами $h_{H}$ и $h_{L}$, где $h_{H}=$ $=0,01 \lambda /\left(4 n_{H}\right)$, причем $h_{L} n_{L}+h_{H} n_{H}=\lambda / 2$, длина волны $\lambda=10$ мкм. Канал потерь для данного селектора представлен выходом пучка из резонатора лазера через брэгговское зеркало. Существенное различие в толщинах слоев $h_{H}$ и $h_{L}$ приводит к узкой полосе в отражении, необходимой для выделения отдельной линии генерации. Расчет показывает, что для достижения коэффициента отражения в резонатор, близкого к $100 \%$ для выделяемой линии, необходимо иметь количество периодов структуры $\sim 10^{3}$; полная толщина составит $10^{3} \lambda /\left(2 n_{L}\right) \approx$ $\approx 2$ мм. Можно также рассмотреть и структуры с модуляцией коэффициента преломления, однако толщина образца будет того же порядка.

Можно рассмотреть еще один вид спектрального селектора в виде наклонного интерферометра, изготовленного, например, из пластины $\mathrm{ZnSe}$ толщиной порядка 10 мкм, имеющей зеркальные покрытия с отражением $R \geq 0,9$ с обеих сторон. Канал потерь для 
подавляемых линий в данном случае - это вывод пучка из резонатора за счет отражения от расстроенного интерферометра. Потери для выделяемой линии генерации состоят из потерь в разделительном слое $\mathrm{ZnSe}$ (остаточное поглощение материала плюс потери на взаимодействие с поверхностью, определяемые технологией изготовления отражательных покрытий) и потерь из-за смещения пучка, в данном случае незначительный из-за малой толщины наклонного интерферометра. Для такого селектора существует дополнительная трудность перестройки по линиям - устройство не может работать в широком спектральном диапазоне. Выходом может служить клиновидная структура, аналогичная той, что была предложена в [8].

Идея использования наклонного интерферометра с воздушным разделительным слоем порядка 10 мкм и с его перестройкой с помощью пьезотранслятора встречает трудности в практической реализации. Устройство в этом случае будет более громоздким по сравнению с представленным выше наклонным интерферометром из клиновидной тонкой пластины. В результате температурные флуктуации устройства не позволят с достаточной точностью управлять базой интерферометра, и, следовательно, выделение требуемой линии генерации также станет нестабильным.

Недостатки присущи также отражательному интерферометру с поглощающей (например, металлической) пленкой в качестве источника потерь для подавляемых линий [9]. В этом случае, чтобы обеспечить необходимое подавление линий генерации, соседних с выделяемой, необходимо нанести с обеих сторон металлической пленки дополнительно по крайней мере по 3 пары диэлектрических слоев (например, $\mathrm{ZnSe} / \mathrm{GaAs}$ ). Потери для выделяемой линии могут быть обусловлены неоднородностью металлической пленки, а также (неизбежной при высокой температуре в процессе изготовления) диффузией металла в соседние диэлектрические слои структуры.

Влияние поглощения, принципиально присутствующего в описанной выше структуре, преодолевается при использовании многолучевого фазового интерферометра [10], представляющего собой решетку из фазовых интерферометров [11; 12], выполненных таким образом, чтобы соседние уча- стки фронта падающей волны для выделяемой линии генерации не нарушали фазового фронта при отражении. При этом для подавляемых участков спектра имеется существенное искажение волнового фронта, приводящее к выходу части падающего пучка из резонатора. Устройства, реализующие этот принцип спектральной селекции, обладают существенными недостатками. Для обеспечения высокой селективности соседние участки покрытия в области выделяемой линии должны иметь максимальное различие в дисперсии фазы $d \Phi / d \lambda$, где $\Phi$ фаза отраженной от участка покрытия волны. При наличии потерь в слоях покрытия это соответствует максимальной амплитудной модуляции по фронту волны, т. е. выводу части пучка из резонатора, и, следовательно, приводит к потерям для выделяемой линии генерации. Неидеальность границ соседних участков покрытия приводит к такому же результату.

Использование обычного интерферометра Майкельсона (со светоделительной пластиной из прозрачного в ИК области спектра материала) лишено смысла, так как выделяемый по уровню 0,7 участок спектра для $\mathrm{CO}_{2}$ лазера охватывает всю спектральную область 9-11 микрон. Канал потерь для подавляемых линий в этом случае определяется выводом пучка из резонатора при отражении от светоделительной пластины.

\section{Интерферометр Майкельсона с внутренним фазовым интерферометром (ИМФИ)}

Предлагаемое устройство интерферометpa (ИМФИ) показано на рис. 1. Оно сочетает в себе свойства фазового интерферометра Жиреса - Турнуа [11] с двухлучевым характером интерферометра Майкельсона, определяющим его канал потерь для подавляемых линий генерации. Поскольку коэффициент отражения в резонатор определяется разностью фаз полей, отраженных от зеркал 3 и 4 (см. рис. 1), то, если выбрать для зеркала 3 участок с большой дисперсией фазы $d \Phi / d \lambda$ в области выделяемой линии, то можно существенно увеличить селективность.

Для примера проведем расчет коэффициента отражения ИМФИ для $\lambda=9,6574$ мкм (линия 9P(32) $\mathrm{CO}_{2}$ лазера). Зеркало 3 состоит из 20 пар четвертьволновых $h_{L}$ слоев 
Puc. 1. Оптические схемы ИМФИ: $a$ - устройство для селекции отдельной линии генерации; 1 - разрядная трубка $\mathrm{CO}_{2}$ лазера; 2 - светоделительная пластинка; 3 - зеркало с фазовым интерферометром (1-е зеркало ИМФИ); 4 - 2-е зеркало ИМФИ; 6 - вариант с перестройкой по спектру генерации и выделением избранной линии генерации; 5,6 - зеркала поворотного устройства
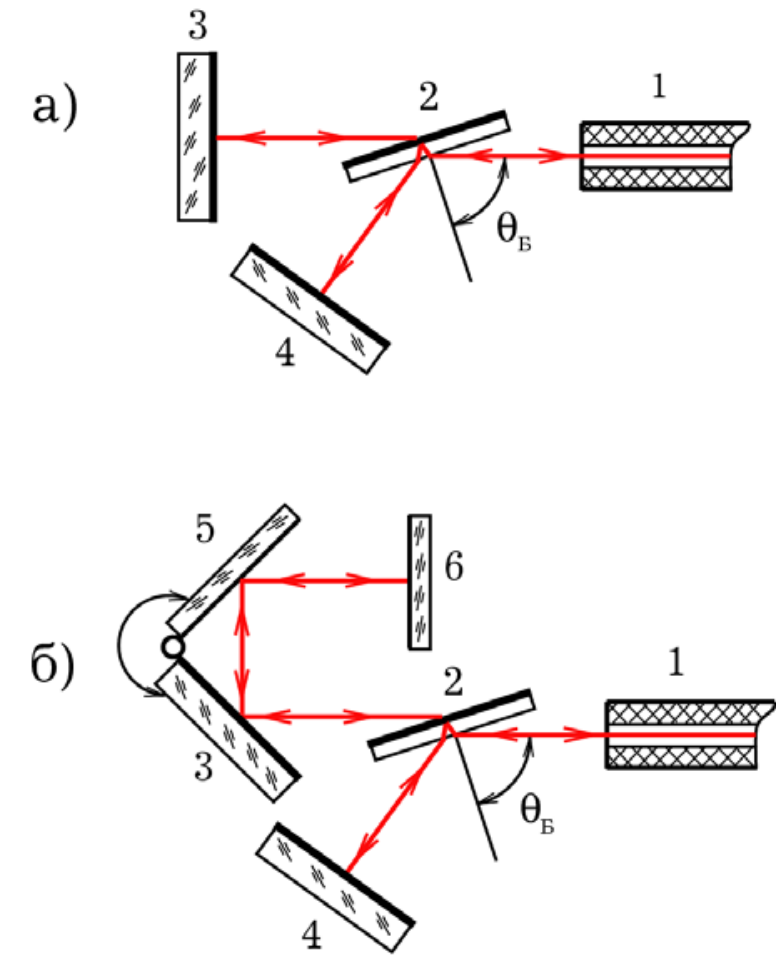

Майкельсона. Необходимую перестройку по линиям можно осуществить только изменением толщины разделительного слоя $h_{w}$. В частности, это может быть реализовано при использовании клинового покрытия. Проведем оценку применимости этого варианта перестройки по линиям генерации для $\mathrm{CO}_{2}$ лазера. Допустим, что мы можем смещать зеркало 3 (см. рис. $1, a$ ) в поперечном (к направлению падения пучка излучения)

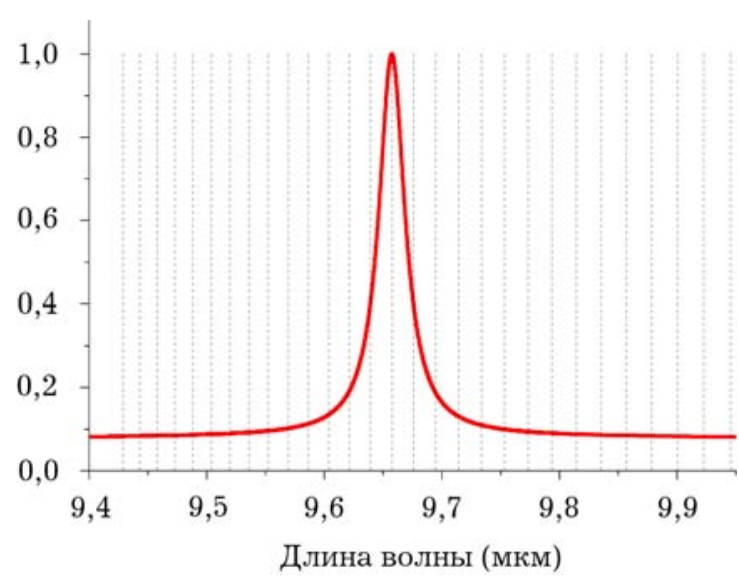

Puc. 2. Спектральная характеристика коэффициента отражения ИМФИ для $\mathrm{CO}_{2}$ лазера вблизи выделяемой линии. Вертикальными штриховыми линиями показаны положения линий генерации этой ветви, соседних с выделяемой 9Р(32) линией 


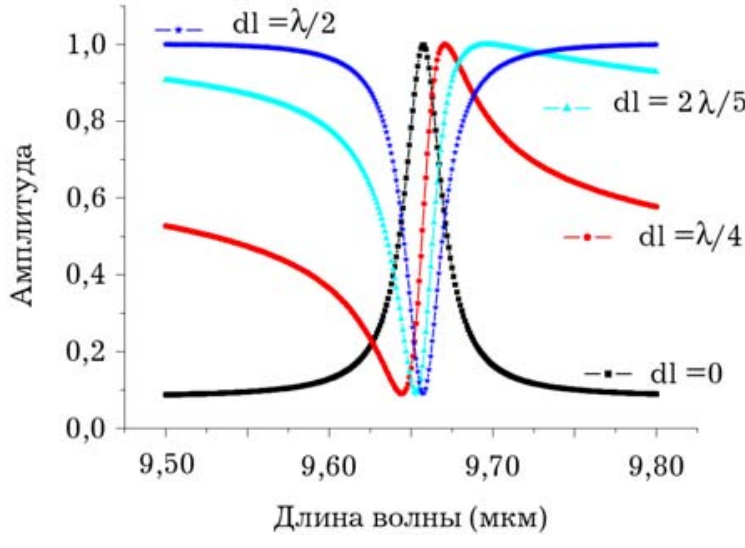

Puc. 3. Зависимость формы спектра коэффициента отражения при смещении одного из зеркал ИМФИ на величину $d l$ относительно точки фазового согласования: $1-d l=0 ; 2-d l=\lambda / 4 ; 3-d l=2 \lambda / 5 ; 4-d l=\lambda / 2$

направлении на расстояние $D=30$ мм; тогда для охвата всей области генерации $\mathrm{CO}_{2}$ лазера от 9 до 11 микрон, порядка 120 линий, необходимо иметь клин $\sim 0,004^{\circ}$. Иначе говоря, на одну линию генерации будет приходиться область покрытия с поперечным размером 0,25 мм, а типичный поперечный размер пучка в волноводном лазере $~ 2$ мм. Для решения этой проблемы возможны два пути. Во-первых, можно использовать несколько селекторов на отдельные группы линий генерации (для $\mathrm{CO}_{2}$ лазера это группы колебательно-вращательных переходов в ветвях 9R, 9P, 10R, 10P). Селекторы могут быть выполнены на одной подложке, с разными клиновыми разделительными слоями $h_{w}$. Переключение между различными селекторами можно осуществлять смещением зеркала 3 (см. рис. 1, a) в направлении, перпендикулярном плоскости рисунка. Во-вторых, можно использовать круговые покрытия, эффективно увеличивающие расстояние $D$ до значения $2 \pi D[8]$.

Другой вариант ИМФИ, показанный на рис. $1, \sigma$, допускает перестройку по линиям, включая выделение избранной линии генерации, путем изменения угла падения пучка излучения на зеркало 3. Ось вращения является линией пересечения плоскостей зеркал 3 и 5, поэтому поворот вокруг нее не меняет положения отраженного от интерферометра пучка в пространстве. Возможная область перестройки здесь также ограничена одной ветвью генерации $\mathrm{CO}_{2}$ лазера. Например, при крайних углах наклона 20 и $70^{\circ}$ и толщине разделительного слоя $h_{w}=10,65$ мкм реализуется диапазон 9,4-10,12 мкм, т. е. полностью покрывается ветвь генерации 9Р $\mathrm{CO}_{2}$ лазера. Зеркало поворотного механизма 5 и зеркало 6 имеют высокий коэффициент отражения, аналогично зеркалу 4. Изменение угла падения на зеркало 5 при перестройке по линиям незначительно влияет на отражательную способность «стола» покрытия даже для показанной на рис. 1 -поляризации лазерного излучения из-за высоких показателей преломления $\mathrm{ZnSe}$ и $\mathrm{GaAs}$. Учитывая двукратное отражение излучения от зеркала 3, количество слоев поверх разделительного слоя может быть уменьшено. Для перестройки во всем диапазоне генерации $\mathrm{CO}_{2}$ лазера зеркало 3 может быть выполнено в виде секций с различными толщинами разделительных слоев $h_{w}$ с переключением между ними в направлении, перпендикулярном плоскости рисунка.

\section{Обсуждение и выводы}

В данной работе проведен критический анализ существующих, а также более или менее возможных к исполнению частотных селекторов линий генерации молекулярных лазеров ИК диапазона спектра.

Предложен вариант селектора на основе модификации интерферометра Майкельсона, в котором одно из зеркал является отражательным фазовым интерферометром ИМФИ. Предложенная технология изготовления ИМФИ представляется вполне доступной к исполнению для современного уровня технологии диэлектрических покрытий. Расчеты показали, что такое устройство способно обеспечить необходимое подавление соседних с выделяемой линий генерации $\mathrm{CO}_{2}$ лазера при малых потерях для выделяемой линии. Предложены два варианта перестройки по линиям генерации при одновременном надежном выделении каждой избранной линии.

\section{Список литературы}

1. Patel C. K. N. Continuous-Wave Laser Action on Vibrational-Rotational Transitions of $\mathrm{CO}_{2} / /$ Physical Review. 1964. Vol. 136 (5A). P. 1187-1193.

2. Yong Zhang, Tim Killeen. Gas Lasers: $\mathrm{CO}_{2}$ Lasers - progressing from a varied past to an application-specific future // Laser Focus World. 2016. 4 November. 
3. Pigeon J. J., Tochitsky S. Ya., Gong C., Josh C. Supercontinuum generation from 2 to $40 \mu \mathrm{m}$ in GaAs pumped by picosecond $\mathrm{CO}_{2}$ laser pulses // Optics Letters. 2014. Vol. 39. P. 3246-3249.

4. Смит П. В. Селекция мод в лазерах // Tp. инженеров по электронике и радиоэлектронике (ТИИЭР). 1972. Т. 60. С. 106-128.

5. Троиџкий Ю. В. Одночастотная генерация в газовых лазерах. Новосибирск: Наука, $1975.159 \mathrm{c}$.

6. Василенко Л. С., Дюба Н. М., Ковалёв $A$. A. Волноводные $\mathrm{CO}_{2}$ лазеры // Перестраиваемые лазеры и их применение: Сб. ст. / Под ред. В. П. Чеботаева; СО АН СССР, Институт теплофизики. Новосибирск, 1988. C. $105-117$.

7. Hologan A. M., Prunty S. L. Line selection in carbon dioxide waveguide lasers using diffraction gratings // Infrared Physics. 1983. Vol. 23. P. 149-156.
8. Thelen A. Circularly Wedged Optical Coatings: Theory // Applied Optics. 1965. Vol. 4. P. 977-985.

9. Захаров М. И., Троицкий Ю. В. Расчет оптического резонатора с селекцией мод за счет поглощения и рассеяния света // Оптика и спектроскопия. 1971. Т. 30. С. 490-495.

10. Троиикий Ю. В. Использование многолучевого фазового интерферометра для получения одночастотной генерации в лазеpax // Квантовая электроника. 1975. Т. 2. C. 2444-2452.

11. Gires F., Tournois P. Interferometre utilizable pour la compression lumineuses modulées en frequence // C.R.Ac.Sci. Paris, 1964. Vol. 258. P. 6112-6115.

Материал поступил в редколлегию 13.02.2018

\author{
A. A. Kovalyov \\ A. V. Rzhanov Institute of Semiconductor Physics SB RAS \\ 13 Academician Lavrentiev Ave., Novosibirsk, 630090, Russian Federation \\ kovalev@isp.nsc.ru
}

\title{
GENERATION LINES SELECTOR FOR MOLECULAR IR LASERS
}

Applicability of several approaches to generation lines selection in molecular IR laser is investigated: diffraction grating placed in spite of one of cavity mirrors, Bragg mirror, inclined and reflective interferometers. A selector is proposed which combines advantages of Michelson interferometer with phase reflective interferometer, and which permits reliable selection of a chosen vibrationrotation generation line. The selector calculation is performed for the case of waveguide $\mathrm{CO}_{2}$ laser. Two variants of schemes for laser tunability over generation lines are analyzed by using this selector.

Keywords: line generation selection in IR molecular lasers, Michelson interferometer, phase reflective interferometer.

\section{For citation:}

Kovalyov A. A. Generation Lines Selector for Molecular IR Lasers. Siberian Journal of Physics, 2018, vol. 13, no. 1, p. 102-107. (In Russ.)

DOI 10.25205/2541-9447-2018-13-1-102-107 